\title{
Chronic purulent nasal discharge
}

This Endgames paper (BMJ 2017;357:j2061, doi:10.1136/bmj. j2061) incorrectly described the location of the fungal ball (or mycetoma) as being in the right nasal cavity. It should have read "in the left maxillary sinus."

The following corrections therefore apply:

1. In the Answer, "A fungal ball, also known as a mycetoma, in the right nasal cavity" should read, "A fungal ball, also known as a mycetoma, in the left maxillary sinus."
2. In the Discussion, "The coronal sinus CT scan shows a fungal ball in the right nasal cavity (fig 2)" should read, "The coronal sinus CT scan shows a fungal ball in the left maxillary sinus (fig 2)," and "The bony wall of the right maxillary sinus is sclerotic" should read, "The bony wall of the left maxillary sinus is sclerotic." 